SHS Web of Conferences 24, 02006 (2016)

DOI: $10.1051 /$ shsconf/20162402006

(C) Owned by the authors, published by EDP Sciences, 2016

\title{
Analysis on the semantic evolution of Chinese Gan dialect from the perspective of cognition
}

\author{
Jiugen Xiao ${ }^{1}$, Xijuan Chen $^{2} \&$ Lingjun Xiong ${ }^{3}$ \\ ${ }^{1}$ Jiangxi Normal University, College of Liberal Arts, Nanchang, Jiangxi, China \\ ${ }^{2}$ Nanchang Institute of Science and Technology, Education Department for Ethnic Minorities, Nanchang, Jiangxi \\ China \\ ${ }^{3}$ Nanchang Institute of Technology, College of Media, Nanchang, Jiangxi, China
}

\begin{abstract}
The meaning of a word is the reflection of social life, and it is the result of the cognition of the object, phenomenon and relationship through the mental activity. The development and changes of lexical meanings essentially reflect the development and change of people's cognitive activities, so people's cognitive awareness is reflected in words, which is also reflected in the word meaning evolution of Chinese Gan dialect. Whether in the extended way or pattern, the linkage, radiation, complex comprehensive changes of the lexical meaning of Gan dialect in Chinese, all follow a rule: The expression of the meaning in cognitive process will correspondingly change with the continuous deepening of human cognition.
\end{abstract}

Keywords: cognitive perspective; Chinese; Gan dialect; lexical meaning evolution; main route

\section{INTRODUCTION}

Cognitive linguistics believes that any element of a language cannot exist independently without the human cognitive activity. Thus, without one's cognitive activities, there is no human language as well as no words to express people's mental activities. Therefore, the relationship between the cognition and meaning of words is very close. Cognition is the foundation of the meaning, and the development of cognition promotes the development and change of the meaning of words; word meaning is the mapping of cognitive activity, the development and changes of lexical meanings also reflects the development and change of human cognitive processes. Studying on people's cognitive activities helps to study language meaning, which also helps to reveal people's cognitive rules, not only in the semantic study of ordinary language, but also in the semantic study of regional dialects.

There is no doubt that the evolution of the meaning of Gan dialect (Jiangxi dialect) also reflects the development of people's cognitive activities. How did the lexical meaning develop and in which way? The following is the explanation and analysis on it from the perspective of the development and changes of the cognitive activity.
It is found in the investigation that the evolution of the meaning of Gan dialect contains two aspects: the development of the meaning and the change of the meaning. The development of the word meaning belongs to the general extending of meaning, that is a word or words produce new meanings, and its original meaning is still in use; the change of the meaning or semantic change means a word or words produce new meanings and, its original meaning disappeared. There are many similarities between these two aspects, so in the discussion of the development and change of word meaning, these two aspects will not be disaggregated, but can be taken as two items of one group with more specific analysis of the differences.

\section{ANALYSIS ON THE WAYS OF THE} EVOLUTION OF THE LEXICAL MEANING FROM THE PERSPECTIVE OF COGNITIVE ACTIVITIES

In the evolution of the meaning of Gan dialect, the extension of word meaning is the main way, which is a kind of regular movement of word meaning. The so-called extended meaning based on the original meaning can produce a new mode of development 


\section{SHS Web of Conferences}

through the association. Here, the "association" is a kind of mental activity of people in cognitive domain, and the mental activities have a close relation with the traditional ethnic culture, which makes the extended meaning often with certain ethnic content. As $\mathrm{Lu}$ Zongda and Wang Ning said, a new word meaning is based on its original meaning along with the direction of its characteristics according to the habits of all ethnic groups, and the new meanings or derived words can be achieved ${ }^{[1]}$. Zhou Guangqing also believes that the extended meaning is a kind of mental activity related to association; as a mental activity, association also lies in the traditional culture and can be aroused, guided and controlled ${ }^{[2]}$.

Then, what is the origin and way of the extended meaning? Here are the explanations.

\subsection{The origin of the extension of the word meaning}

Any change of things stem from their origin, like water stems from its source and trees from their roots. The extended meaning is no exception. The origin is the original meaning of the word and the source of the extended meaning of the word. Therefore, to grasp the extension of the meaning, people should first find its origin.

In the development and change of word meaning, some of the original meaning of the word is the basic meaning which is commonly used, and its development is relatively clear. But in the process of development of some of the words, the original meaning has been less frequently or not been used, so it becomes difficult to find the development and changes of the meaning clearly. In this case, to clarify the origin of the meaning of the word, people have to resort to the history of the literature. For example, in Gan dialect area, people often take "inform" or "convey (some kind of news, etc.)" as "Bao", here it is the extended meaning. So, what is the original meaning? How did the meaning come from? People should seek out the answers from the literature. In the book Shuowen Qianbu: "Bao, means Dang the sinners" [3]. Cui Hao gives the note that "Dang, means punish." Obviously, "Bao" originally means the judgment of crimes. An article of an ancient Chinese classic Han Feizi, $W u \mathrm{Du}$ says: "There is a man Zhigong in Chu Dynasty. His father stole a sheep and he went to inform his father to the officer. The officer said, 'to sentence to death.' It shows that Zhigong is loyal to the king but betray his father, finally Bao to death." "Bao" is used in the original meaning, meaning "judgment". The ancient judgments of sinners generally have to be reported, so this is extended to "report" to the higher level. Shiji, Huajizhuang, "It is my fault, so I should bother you to Bao the river man." At the beginning, the report means to convey the bad news, and later both good and bad news can be reported. For example, Down in Mingfa Port by Yang Wanli in Song Dynasty notes, "In dawn, the pole man Bao for sending the vessel." Xixianji by Wang Shifu in Yuan Dynasty notes, "Remember the visitors, when master came back, Bao him." In Gan dialect, "Bao" focuses on the bad news, such as in funeral etc. Therefore, it is clear to find that the development of the meaning of "Bao" can be concluded like this: the judgment of the crimes $\rightarrow$ report to (the higher) $\rightarrow$ inform or convey (a message, etc.).

Meaning changes along with the constantly deepening cognition of people, and "association" in cognitive domain is the bridge leading to the extended meanings from the original meaning of the word. So the original meaning is a source for cognition and the starting point of the extended meaning.

\subsection{The way of extended meaning}

Because people have the deepen cognition to the world and the change of the meaning of the word is also increasingly complex, many words have two or more meanings, which form the complex network of extended meanings. In Gan dialect, there are also many words that have complicated extended meaning relations. As far as the way of extension, there are several cases as follows.

1. Chain extension

The original meaning A can be extended to the meaning B. Similarly, B also can be extended to a new meaning $\mathrm{C}$, and $\mathrm{C}$ is extended to $\mathrm{D}$... As a result, all the extended meanings form a chain line beginning with A, which can be called chain extension. Those extended meanings are different with the relations between A.

Such examples in Gan dialect are common. For example, "Biao" means "running dogs" in the begingning. In the book Shuowen, Quanbu says: "Biao refers to running dogs" ${ }^{[3]}$. An ancient Chinese collection of poetry Chuci, Jiubian says: "what a flood of clouds, biao running under the moon!" Hong Xingzu in Song Dynasty notes that "Biao refers to the appearance of dog running" ${ }^{[4]}$. From the above examples, its original meaning is extended to "running". Duan Yucai notes that "Biao is extended to run for anyone" ${ }^{[5]}$. In ancient Chinese collection of poetry Chuci, Yunzhongjun: "Cloud God drops in the earth and biao in a while". And Wang Yizhang notes that "Biao means dashing in appearance" [4]. Thus, the meaning "running" also changes to "fast of liquid injection". Yongyanjigu by Ying Zhong notes that "water would biao like an arrow when carrying the pipe with powe" ${ }^{[6]}$. In the regions of Ji'an, Jishui, Anfu, Xiajiang in Jiangxi province, people use the meaning "running", both referring to people and animals, such as, "qu (he) biao (run) really fast"; they also use meaning "injection", such as "open the lid, the beer biao (inject)." The diagram for the development of its word meaning is:

A. $\operatorname{dog}$ runs $\rightarrow$ B. (person or other animals) running $\rightarrow C$. (liquid) jet 
The original meaning of "Yi" is "hard work". The famous ancient Chinese classic Erya, Shigu says: "Yi refers to hard word" "[7]. In the Book of Songs, Xiaoya, Yuwuzheng says: "he left from home, but did not know my Yi." And Maozhuan says: "Yi refers to labor." Then, the meaning is extended from hard work to "the artifacts being impaired". Duan Yucai notes that "Artifacts in a long time use with the impaired is called Yi. Yang Shen in Ming Dynasty said that 'Yu' was used to describe the worn artifacts, according to the survey, not Yu but $\mathrm{Yi}$ " "[5]. Cities such as Nanchang City, Xiushui City or other cities in the northwest of Jiangxi Province, people use the meaning "worn artifacts", such as "the screw Yi ". The diagram of the development of its word meaning is:

A. (person) long and hard work $\rightarrow$ B. (artifacts) wear and tear after a long time

From the examples, this kind of meaning is gradually extended with the development of people's cognitive activities. And the extension is characterized by the sense of meaning moving in a direction of development, as shown in the diagram:

$$
\mathrm{A} \rightarrow \mathrm{B}, \mathrm{B} \rightarrow \mathrm{C}, \mathrm{C} \rightarrow \mathrm{D} \ldots \ldots
$$

\section{Radiation extension}

The original meaning A with multiple meanings that can be used to extend to a number of new meanings of the parallel relationship of B, C, D...This forms an expanding network centered at A. There is a direct relationship between each word meaning and the extended meaning.

There are a lot of cases of the extension in Gan dialect. For example, the meaning of "Housheng" extends to a number of extended meanings in parallel relations The original meaning is "birth afterwards". The famous ancient Chinese classic Erya, Shiqin says that "the man who was the firstborn is the elder brother, and one born afterwards is the young brother" ${ }^{[7]}$. Thus, one of the extended meanings is "descendants". For example, in the Book of Songs, Shangsong, Yinwu: "live long and healthy to protect our descendants." Zheng Xuan notes: "King can live long and be healthy to protect our Housheng (descendants)." The second extended meaning is "next generation". For example, Analects of Confucius, Zihan notes, "we can make good expectation on Housheng (the next generation), and they may be better than us." The third is "the young man". The ancient novel Zhang Daoling Tests Zhaosheng for Seven Times says: "The priest is more than 60 years old in reality. However, he turns to be young as Housheng (young) as thirty in appearance after taking precious medicine". Therefore, the fourth means "young". In Liexian zhuan (Biographies of Immortals), "keep the essence of energy and spirit, then white hair can turn to black and the tooth can be fresh and young", cited by Li Xian. The fifth is "afterlife", namely the second life. Family Motto of Yanshi by Yan Zhitui in Qi Dynasty, "the Housheng (second life) is the retribution of the current life." The diagram of the development of its word meaning is:

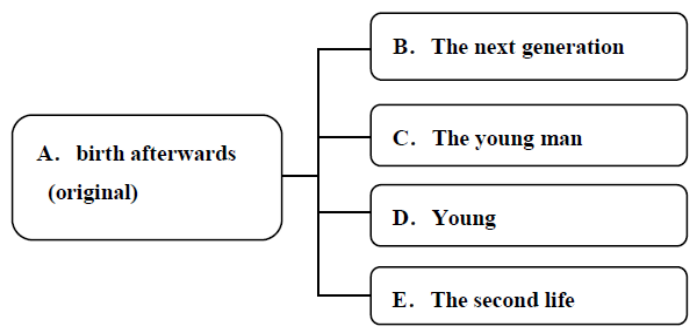

These meanings in Gan dialect areas are all commonly used, especially the "young" meaning for the most, which often appears in the speaking of countrywomen, and even the old man never read can use it.

Another example "Xiao" is commonly used in Gan dialect area, whose original meaning is "bright". In Shuowen Ribu: "Xiao refers to bright" [3]. In Chuang-tzu, Tiandi: "invisible but only can see Xiao (bright)". Several meanings are derived from its original meaning. The first one is "dawn". Duan Yucai notes that "it is also called it is Xiao (dawn) outside" [5]. An ancient Chinese historian Sima Zhen once said: "When the cock crows for three times, it is Xiao (dawn) outside." In Yiliu region of Gan dialect area, people still use the meaning "bright" or "dawn", such as the saying in Yichun: "It is Xiao (bright)!" The second extended meaning is "understand". Volume 1 of Dialects notes that "Xiao is known as understanding"[8]. Qian Yijian in Qing Dynasty notes that "Xiao refers to understanding in volume thirteen....In Travel Notes of Tiantai Mountain by Sun Chuo: "People cannot see the road clearly, so they do not Xiao (know) the way." "[9] The third meaning is to "enlighten". In Zhinangbu, Sun Zhongchun by Feng Menglong in Ming Dynasty: "Sun Xiao (enlightened and told) them and said: 'You do not make a future plan...Salt not sold, left a disaster in thirty years later.' "The Fourth is "master" or "be proficient (some skills)". In the history book Sui Shu, Biography of Wang Chong in Sui: "(Wang Chong) is fond of the art of war and Xiao (be good at) strategies." The double syllable "Xiaode" is commonly used in daily life of Gan dialect area. The diagram of the development of its word meaning is:

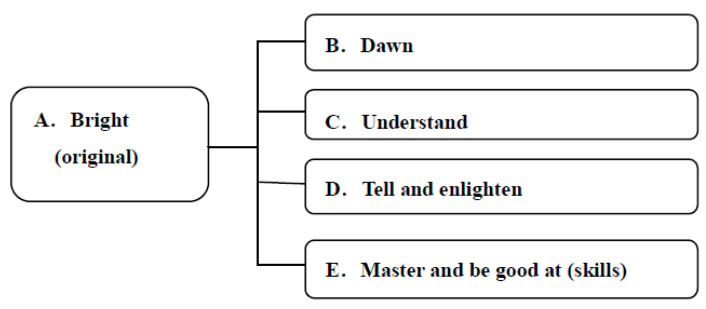

The feature of the radiation extension is shown as follows: 


\section{SHS Web of Conferences}

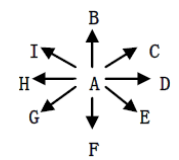

Each extended meaning is derived from the original meaning and the extension is like the radiation to all rounds.

Cognitive linguistics believes that the cognitive psychological mechanism of human can influence the development and change of word meaning, which is the universal law of various languages. From the above examples, it can be seen that the derivation of the meaning of the word is the multi distribution. This shows that the mental imagery activity of human is multi-dimensional, and the image form of its cognition is also multi-dimensional like the radiation.

3. Complex synthesis extension

In the process of lexical development, the extensions are not always single. Sometimes chain extension and radiation extension appear at the same time to the meanings of the same word, forming the complex relationship of multiple levels and interactive extensions, therefore it is known as complex synthesis extension.

The extension is not uncommon in Gan dialect. For example, "Min" originally means "fishing thread". In Shuowen Mibu: "People can use the Min (fishing thread) for the clothes." [3] In Poetry, Zhaonan, Hebi Nongyi: "How to fish? Use Min (fishing thread)." Gao Heng notes that "Min refers to fishing rope." Thus, Min originally means fishing thread. In Send to Cheng by $\mathrm{Han} \mathrm{Yu}$ in Tang Dynasty: "Picking fern in the mountains, fishing with Min." The extended meaning from the original meaning is the common "rope". For example, in Liunan Essay by Wang Yingkui in Qing Dynasty: "The book room and kitchen are only separated by curtain which is suspended and controlled by Min (rope)." Due to rope is used to thread money, and then Min refers to "money rope". And in Guangyun, Zhenyun: "Min is used for money rope." [10] This meaning can be also found in Book of Han, Wudi: "count the Min (strings) of money." Li Fei notes that "Min, a rope, is used to thread the money." Then the extended meaning becomes "strings of coins". In The old History of the Five Dynasties, Han, Wang Chuan: "The unit of Min (strings of money) from official bank is eighty." And then, it becomes "thread money with strings". What's more, there is another example in Songyan, Qiyi by Xu Shiluan in Qing Dynasty: "The old woman sells porridge, and Min (collect the money with strings)." In the regions of Gan dialect, such as Jiangxi Province, and Pingjiang County of Hunan Province, people still use the meaning "collect money with strings" and "strings of coins", with the pronounce "Min" or "Minqian". Even now, people still keep hiding a lot of strings of coins, and the villagers call it "Min ball" or "money ball". With the compre- hensive overview of the development, it's diagram shown as follows:

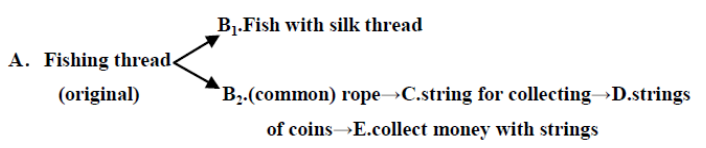

Another example "Shi" means "rice". In Shuowen Shibu: "Shi refers to rice." [3] A famous ancient Chinese Duan Yucai notes that "rice' means 'various rice', which refers to the rice all the people have" ${ }^{[5]}$. In Zhouli, Temple, Kashiwa: "Kashiwa is in charge of King's meal." Zheng Xuan notes that "Shi is rice". Later, the original meaning is extended to "Jiagu (millet)". In Shiji, Weifeng: "The woman has not had Shi for three years." Zheng Xuan also said: "Shi refers to special rice called Jiagu." Then, the meaning changes to "food which is edible". In New Yuefu, Maitan by Bai Juyi: "What does the money use for? Clothing and food." "Food" is edible for person or other animals, and then it also refers to "eating food". In Ancient Yunhui, Zhiyun: "Shi means to eat and taste." ${ }^{[11]}$ At the same time, it can also extend to suck or drink liquid things. In Zhengzitong, Shibu: "Drinding is also called Shi." ${ }^{[12]}$ And in Song Yingxing's Heavenly Creations in Ming Dynasty: "The seedlings growing need Shi (drink) water of three buckets in the day, and five buckets at night to avoid wither." In Gan dialect areas, people not only use the meaning "food" or "rice", but also the meaning "eat", "drink" and "suck" in Fuguang and Guangchang. Thus this meaning can be used whether the food is solid (such as rice and fruit) or liquid (such as water, tea and liquor) or gas (such as smoke, etc.). And the diagram of the word meaning can be shown as follows:

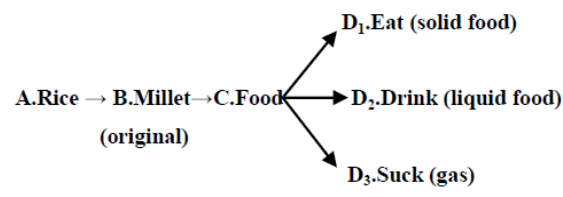

The feature of complex synthesis extension is that chain extension and radiation extension are connected together, which contain complex network for lexical extension. The following is one of the common patterns of the complex synthesis extension. Actually, there are various patterns for the extension.

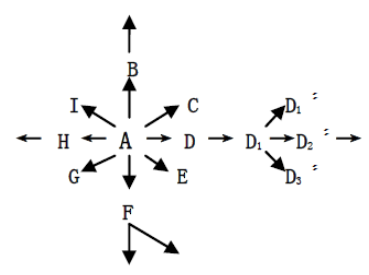

Cognitive linguistics believes that different images 
will be formed in different people's brains toward the same object due to the different cognitive approaches or perspectives. And these different images will cause different forms of development and changes for the meaning. The above examples also show that the development of word complex synthesis extension is not only a representation of human cognition, but more importantly, it can reveal the rules of the development of human cognitive image. The image always reflects in the results of the corresponding cognition. People can seek for the deep motivation for the development of word meaning, and pay attention to the form of the dynamic word meaning ${ }^{[13]}$.

\section{CONCLUSION}

Language emerges in the process of people's cognition, and changes in the process of communication. Cognition is the basis of language formation, and the use of language is the driving force of language development. Language can reflect the social life by the cognitive activities of the material world and the spiritual world. Thus, language is a product of people's cognition, and the word meanings are the reflection of people's cognition in language. At any time, the development of lexical meaning will deeply engraved with the imprinting of human cognitive activities, so the word meaning is also the product of the development of human cognitive activities.

The reflection of the word meaning to social life is essentially the result of the abstraction of the cognition on the thing by the mental activity. The development of word meaning reflects the change of human's cognition to the world, and reflects the human's cognitive ability to the world, and it is a reflection of human's cognition development and change on word meaning. Furthermore, the development of word meaning in Gan dialect truly reflects people's cognition on complex internal and external world in Gan dialect district, and gets a reflection of the deepening of people's cognition.

\section{ACKNOWLEDGEMENT}

This paper is financially supported by China National Social Science Fund Post Funded Projects "Analysis on Origin and Theory of Gan Dialect Archaism "(GN: 14FYY027), Key Projects of Jiangxi Provincial Academy of Social Sciences Joint Planning "Gan Po Dialect and Folk Culture Research" (GN: 13YY01) and Jiangxi College of Humanities and social sciences plan project "Cognitive Analysis on Word Meaning of Chinese Dialect from the perspective of Linguistics" (GN: YY1105).

\section{REFERENCES}

[1] Lu Zongda \& Wang Ning. 1994. Exegetical and Exegesis. Taiyuan: Shanxi Education Press.

[2] Zhou Guangqing. 1992. The cultural psychology on Chinese extension. Journal of Huazhong Normal University, (5).

[3] Xu Shen. 2001. Shuo Wen Jie Zi. Nanjing: Jiangsu Ancient Books Press.

[4] Wang Yi \& Hong Xingzu. 2005. Adittional Notes for Chu Ci Zhang Ju. Changchun: Jilin People's Publishing House.

[5] Duan Yucai. 2002. Annotation for ShuoWenJieZi. Hangzhou: Zhejiang Ancient Books Press.

[6] Ying Zhong. Yongyan Jigu. Manuscripts in Tianyi Pavilion in Ningbo.

[7] Guo Pu, \& Xing Bing. 2010. Annotation for ErYa Shanghai: Shanghai Ancient Books Press.

[8] Yang Xiong. 1985. Dialect. Beijing: Zhonghua Book Company.

[9] Qian Yi. 1984. Notes for Dialec (I). Shanghai: Shanghai Ancient Books Press,

[10] Chen Pengnian. 1983. Jusong Guangyun. Shanghai: Shanghai Ancient Books Press.

[11] Xiong Zhong. 1883. Outline of Ancient Yunhui. Huainan Press in the nine years of the Qing Dynasty.

[12] Zhang Zilie. 1996. Zhengzitong. Beijing: China Workers Press.

[13] Wei Huiping. 2005. Research on the Development of Semantic Development of Chinese Language. Hohhot: Inner Mongolia People's Press, 